Matthieu RAUCH ${ }^{1 *}$

Jorge PIEDRA DORADO ${ }^{1,2}$

Jean-Yves HASCOET ${ }^{1,2}$

Guillaume RUCKERT ${ }^{3,2}$

\title{
A NOVEL METHOD FOR ADDITIVE MANUFACTURING OF COMPLEX SHAPE CURVED PARTS BY USING VARIABLE HEIGHT LAYERS
}

\begin{abstract}
The Wire Arc Additive Manufacturing process (WAAM) is designed for the manufacture of large metallic parts with no joints, very little waste material and hardly any support. It is gaining its space inside the naval, aeronautics and space industries. However, there are key challenges to be solved in order to increase the performance of the WAAM process. Parts with curved shapes are difficult to manufacture with regular parallel layers without support because of an excessive overhang in certain regions. This paper proposes a methodology that solves this issue, by using incrementally angled layers with variable bead height, which eliminates or decreases the overhang between layers. This solution uses an angled rotary positioner (or other method for moving the part in a controlled way) and controls key parameters like the travel speed, the deposition angle, the available bead height difference, etc. The efficiency of the developed proposal is shown with the manufacture of a large curved steel (316L) piece as a use-case.
\end{abstract}

\section{INTRODUCTION}

Additive Manufacturing (AM) is a technique where structures are produced by adding and depositing material in a layer-by-layer manner [1, 2]. Wire Arc Additive Manufacturing (WAAM from now on) is a metallic additive manufacturing process that uses the gas metal arc welding procedure (GMAW) controlled by a CNC machine or robot to create 3D objects in a layer-by-layer strategy. In WAAM, there is an addition of material made with a controlled metallic wire feed, a heat source in the form of a controlled electric arc and a protective shielding in the form of an inert gas atmosphere. Using a multi-axis motion stage, the WAAM process is essentially a three-dimensional welding operation [3]. This process can be used to obtain pieces of any of the wide range of materials compatible with the GMAW process [4] (stainless steel, nickel-based alloys, titanium alloys and aluminium alloys, etc.).

\footnotetext{
${ }^{1}$ GeM - UMR CNRS 6183, Centrale Nantes, France

${ }^{2}$ Additive Manufacturing Group, Joint Laboratory of Marine Technology (JLMT) Centrale Nantes - Naval Group, France

${ }^{3}$ Naval Group Research, Technocampus Ocean, France

*E-mail: Matthieu.Rauch@ec-nantes.fr https://doi.org/10.36897/jme/138820
} 
The deposition rate of the WAAM process is in the magnitude order of the kilograms per hour (among the highest of the additive manufacturing methods) [1,2], which means that WAAM could be the key to economically sustainable manufacturing of large parts.

In this paper, it is provided a new methodology to manufacture complex parts with the wire arc additive manufacturing process. Specifically, the presented research focuses on the manufacture of curved, hollow, single-cordon walled, curved piece of big dimensions made with variable bead height layers. A research about the State of the Art of this topic and its general framework was carried out:

Concerning the toolpath generation, some new solutions and approaches are needed in order to create a toolpath that is compatible with the characteristics of the variable height layers, the traditional 2.5D toolpath generation methods are not suitable for this task. Donghong Ding et al. [5] presented an algorithm to automatically generate optimal tool-paths for the WAAM process for a large class of geometries, which decomposes 2D geometries into a set of convex polygons based on a divide-and-conquer strategy. Isa et al. [6] suggested and developed a new 5-axis path planning model for freeform parts that takes into account the surface profiles of the part. Hascoët et al. [7] proposed a methodology to generate 5 axis toolpaths for WAAM and highlight the main parameters and compared 3 axis and 5 axis toolpaths on part accuracy. Muller et al. [8] proposed an evaluation of toolpaths for additive manufacturing of functionally graded materials (FGM) parts to ensure the manufacturing of parts in compliance with the desired material distribution. About the physics modelling of the process, Ogino et al. [9] created a numerical simulation of WAAM process by a GMAW weld pool model. Ding et al. [10] created a computationally efficient finite element model of WAAM by determining the residual stresses in function of the peak temperatures experienced during the thermal cycles of the WAAM process. About the microstructure, mechanical and chemical properties of the manufactured parts, Dinovitzer et al. [11] discussed the effects of process parameters (travel speed, wire feed rate, current, and argon flow rate) in TIG based WAAM on the bead geometry and microstructure using Hastelloy X alloy welding wire. Rafieazad et al. [12] presented a study of the microstructural (phases fractions and its orientations) and mechanical properties evolution throughout the different regions of low-carbon low-alloy steel parts manufactured with WAAM of low-alloy steel parts. About specific related scientific issues, Lei et al. [13] investigated the humping phenomenon for the multi-directional robotic WAAM. Lam et al. [14] presented an alternative approach to overcome the need of using a rotary table for the fabrication of thin-walled components with overhang features under flat-position deposition conditions by implementing adaptive process control. Yili et al. [15] presented a methodology to manufacture high-building multidirectional pipe joints with WAAM by using the outer cylinder surface of the main pipe to slice other forming pipes of a 10-directional pipe joint. Chen et al. [16] created a WAAM system based on the Cold Metal Transfer (CMT) strategy. McNeil et al. [17] provided a framework for Large Scale Additive Metals Manufacturing (in which WAAM is present) in arbitrary directions by segmenting more complex geometrical parts into gravity aligned, nongravity aligned, and transition segments to help generate toolpaths. Concerning the different manufacturing process comparison and selection, Satish Kumar et al. [18] published a review on WAAM fabricated components of Ti6AL4V and Steels by covering the mechanical properties of WAAM fabricated components, optimal parameters, simulated methods 
of reducing the heat accumulation, grain refinement processes and the heat treatments for fabricated components for the required microstructures. Knezović and Topić [19] presented an explanation of WAAM in detail, gave a view about researches so far in this area and gave suggestions for new advances. Gao et al. [3] discussed the status, challenges, and future of additive manufacturing in engineering, presenting the current barriers, findings, and future trends that are significant to the researchers.

There is one specific scientific paper that deals partially with the same topic as this document, the Vincent Querard PhD thesis [4]. One of the researched topics of this document is the manufacturing of curved parts with WAAM in aluminium. The different approaches studied to manufacture curved parts are:

- Divide the part in sections of constant height layers (Fig. 1, left). Dividing the part in sections increases the risk of voids (in the joint between sections) and creates surface finishing inconsistency.

- Manufacture the part undivided with variable height layers (Fig. 1, centre). This method resulted sometimes insufficient due to the fact that the available bead height difference range is not enough to follow the curve of the part. A part with little radius of curvature and/or big radial section size may not be suitable for this manufacturing approach.

- Manufacture the part with variable height layers and open layers (Fig. 1, right). The open layers compensate the insufficient bead height difference by skipping deposition in those points where the necessary bead height is lower than the minimum bead height possible with the manufacturing process. This solution creates a discontinuity on the material deposition that translates into the layers being off level.
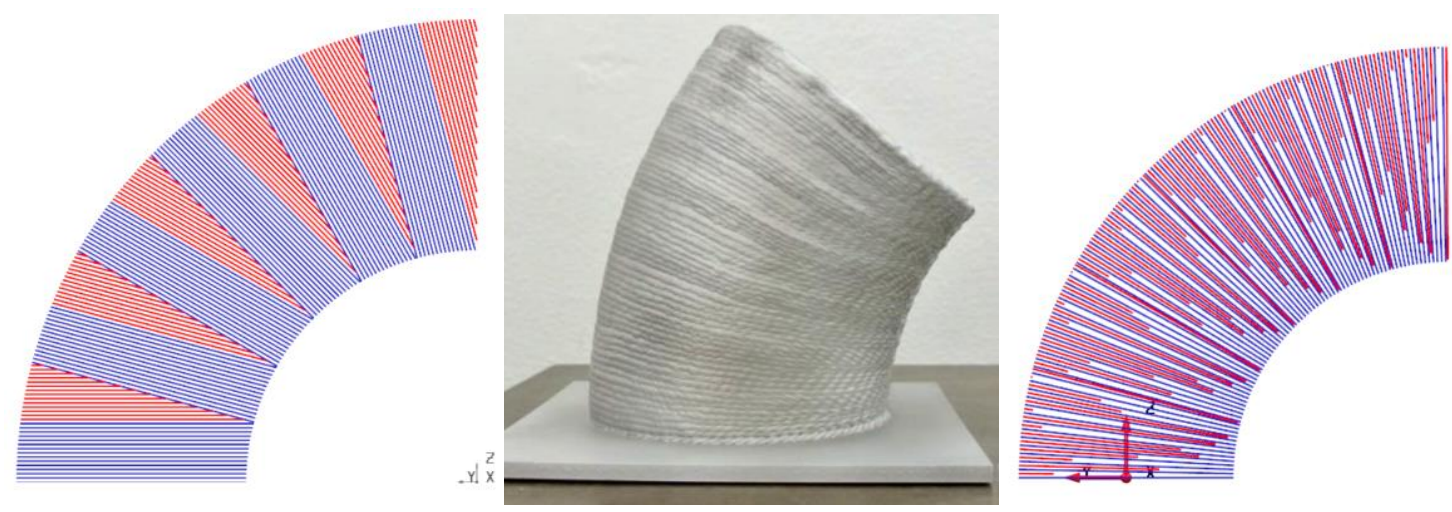

Fig. 1 Past approaches [4]

These solutions shown not to be enough for industrial applications due to the explained problems. Moreover, the metal used is not the same than in the presented paper (incompatible process parameters).

To summarize, in the current state of the research there are already several works done in the subjects of the control of the bead geometry and deposition quality, simulation of the additive manufacturing process, characterization of the chemical and mechanical properties of the manufactured parts and many specific problems concerning the additive manufacturing 
of metallic parts. However, there is very little research about the specific problem (or similar) to be approached in this paper and the only specific research is not suitable for industrial applications jet. The state of the art concerning the toolpath generation does not show any viable solution for the present problem of creating toolpaths compatible with variable height layers. Thus, the present paper will cover the missing research shown above and will use the available information to streamline the research process.

\section{OBJECTIVES OF THE PAPER}

The main objectives of this paper are, the study of the viability to manufacture complex shape, hollow, single cordon wall curved parts made of stainless steel with the WAAM process. Solve the scientific challenge of the manufacture of a curved part (induced overhang) with WAAM without the possibility of the usage of support material. Define a new manufacturing methodology that allows the creation of any generalist part of said characteristics and search for the best geometrical accuracy possible. The final methodology must be as automatic and industrially viable as possible. Verify the final methodology with the manufacturing of a final demonstrator part inside the available time and resources. The study of the mechanical and chemical properties of the manufactured parts is considered to be covered in the past researches and is let out of the scope of this paper.

In order to fulfil the main objectives, the chosen approach is inspired in the second Querard's approach [4]; create variable thickness layers than can grow following the curved shape of the part while maintaining the gravity acceleration perpendicular to the material deposition plane, this approach was chosen because it has the potential to achieve the best surface finish and consistency due to the fact that all layers are complete and no sectioning is carried out. In order to apply this approach, some scientific and technical sub-objectives to meet are proposed:

- Create a regression model of the bead height in function of the process parameters: the proposed approach is to control the bead height with one or more of parameters that most influence it (learned in the state-of-the-art research phase), the wire speed, the tool/travel speed and/or the electric arc power. Instead of using the models reached in other paper, it is chosen to create a new one based on a new experimentation in order to maximize the accuracy of the regression model for the specific WAAM process used.

- Find a solution for the limited range of bead heights: in order to give a more general method that is able to manufacture any parts, no matter its curvature radius size. Also, all the problems and limitations shown in the past approaches should be avoided to the extent possible.

- Achieve the various technical sub-objectives inherent in the constraints produced by the main objectives: Create a correct toolpath definition method: the toolpath has to follow the desired curvature and has to be compatible with the variable bead heights. For that, the initial idea is to slice the part with incremented angled planes through the axis of curvature of the part. Automatic process parameters calculation and definition: it is needed to find a way to calculate and define the different process 
parameters in the final G-Code. Manufacturing of the final demonstrator part. For this, the initial idea is to use a rotary table positioner to manufacture the part while the material deposition is done perpendicularly to the ground. This strategy is chosen due to the fact that it assures that the deposition is always taking place with the wire and the contact normal aligned with the gravity attraction.

The resources available to fulfil these objectives are: a GMAW automatic welding machine, 316L steel wire coils, a KUKA KR500 with a rotary table positioner, a protective and ventilated cage, argon inert gas, a 3D optical scanner and CAD, CAM and numerical computing software.

Following the objectives and sub-objectives above, it is shown below in the Fig. 2 a graphical summary of the suggested manufacturing method of a curved, single-cordon wall, variable bead height part.

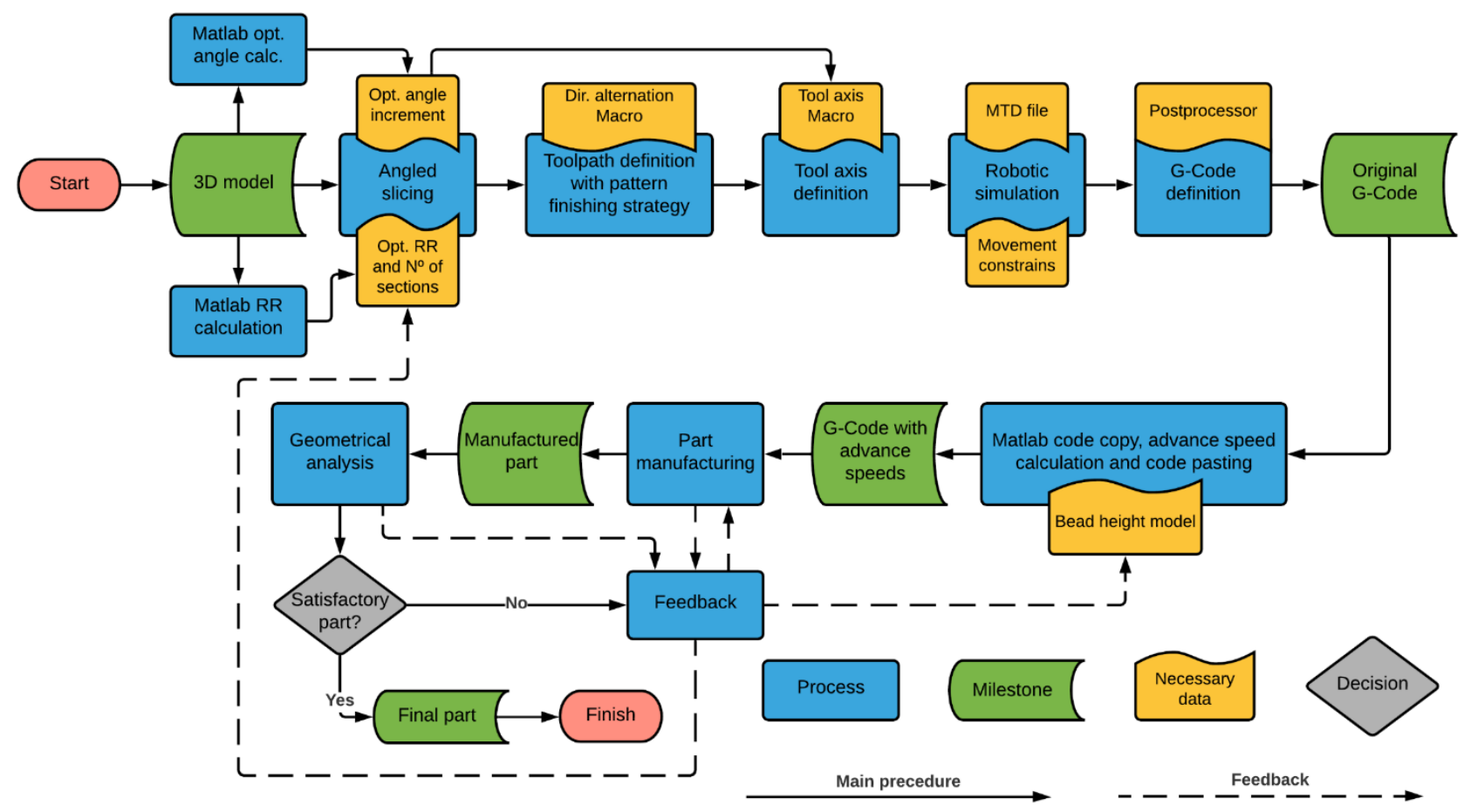

Fig. 2. Procedure summary

\section{BEAD HEIGHT REGRESSION MODEL}

In order to use a variable bead height layer manufacturing process, it has been decided to set an accurate model that can output the correct process parameter for each desired bead height. That model has to be specific to the material and hardware used in the manufacturing process so, an experiment was planned and carried out to observe the bead height in function of the different process parameters. For this, a single variable regression model (Fig. 3) was made by controlling the travel speed of the tool (because it is the easiest and more independent parameter to control). The chosen parameters for the experiment were 5 different travel speeds 
(Ts) with a range of values of 400 to $1200 \mathrm{~mm} / \mathrm{min}$ with a fixed value for the wire speed (WFS) of $8.1 \mathrm{~m} / \mathrm{min}$.

The deposition passes for the experiment were created on top of a previous constantheight layer. This was done to recreate the thermal effects of depositing on top of another layer, moreover, if the deposition is done on top of the substrate, some instability problems could appear earlier in the fastest passes due to the heat sink effect produced by the metal mass of the substrate.

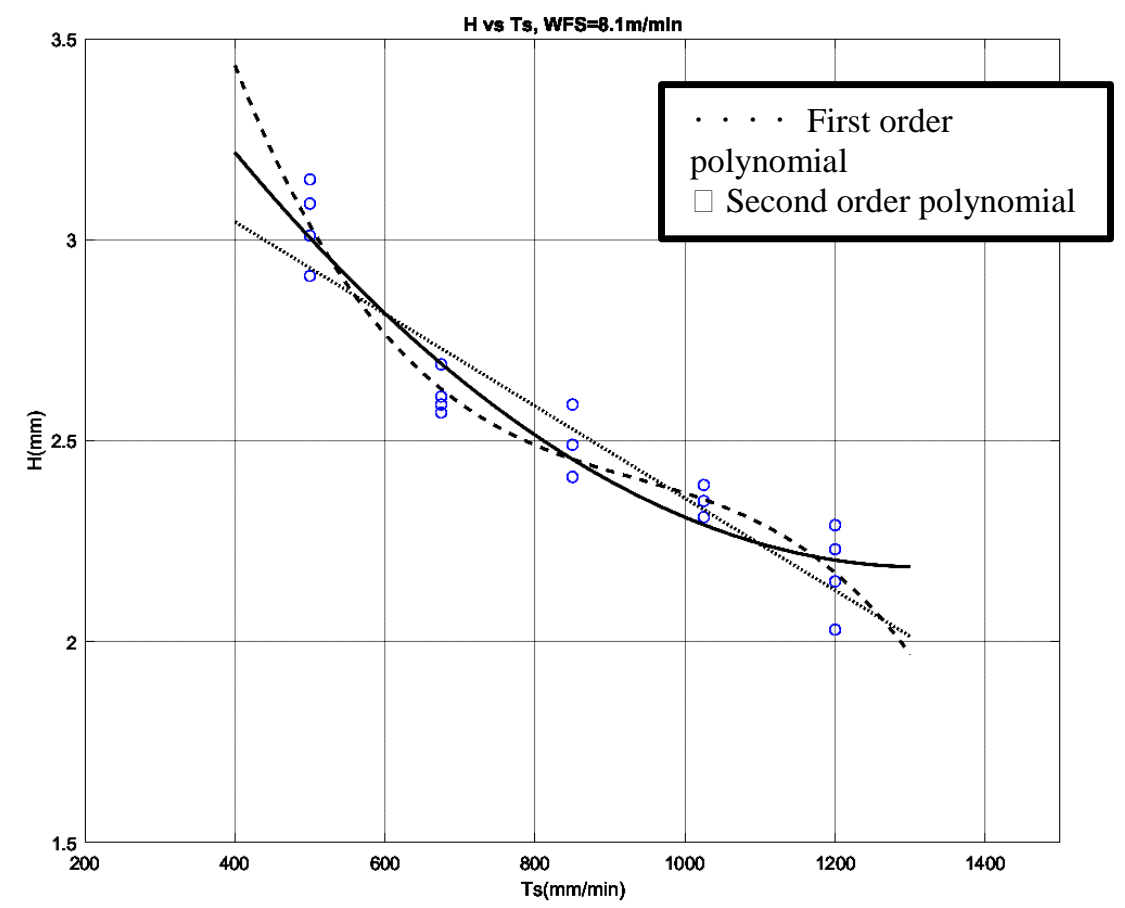

Fig. 3. Bead height $(\mathrm{H})$ vs $T s$, WFS=8.1 m/min. First, second and third order polynomial regression models

The regression model (equation 1) was chosen to be a second order polynomial model since the fitting of the first order model was much lower and the one from the third order was not much higher than the second order one (indicating overfitting). Moreover, the second order polynomial is vastly suggested in the state-of-the-art scientific papers:

$$
\mathrm{H}=1.2245 \mathrm{e}^{-6} \times \mathrm{Ts}^{2}-0.0032 \times \mathrm{Ts}+4.3126
$$

The adjusted $R 2$ of the model is 0.9098 . The admissible range goes from $H=3.2 \mathrm{~mm}$ with $T s=400 \mathrm{~mm} / \mathrm{min}$ to $H=2.2 \mathrm{~mm}$ with $T s=1200 \mathrm{~mm} / \mathrm{min}$. The mean values and standard deviations of the heights measurements are (Table 1):

Table 1. Mean values and standard deviation of the bead heights $(H)$

\begin{tabular}{|l|c|c|c|c|c|}
\hline Travel Speed, Ts $(\mathrm{mm} / \mathrm{min})$ & 500 & 675 & 850 & 1025 & 1200 \\
\hline Mean $H(\mathrm{~mm})$ & 3.04 & 2.61 & 2.49 & 2.34 & 2.17 \\
\hline SD of $H(\mathrm{~mm})$ & \pm 0.06 & \pm 0.03 & \pm 0.04 & \pm 0.02 & \pm 0.06 \\
\hline
\end{tabular}




\section{BEAD HEIGHT RANGE LIMITATIONS}

Due to the limits of the maximum and minimum value of the bead height, not any curved part can be done with the initial chosen approach. There is a limit when the radial size of the sections is too big, or the radius of rotation is too small. Outside that limit, the required difference in bead height between the furthest and closest points from the centre of rotation will be outside the available bead height range of the model. A ratio was created to quantify the feasibility of manufacturing the parts using this procedure; it is called the curvature-size ratio (CSratio, Fig. 4 and equation 2):

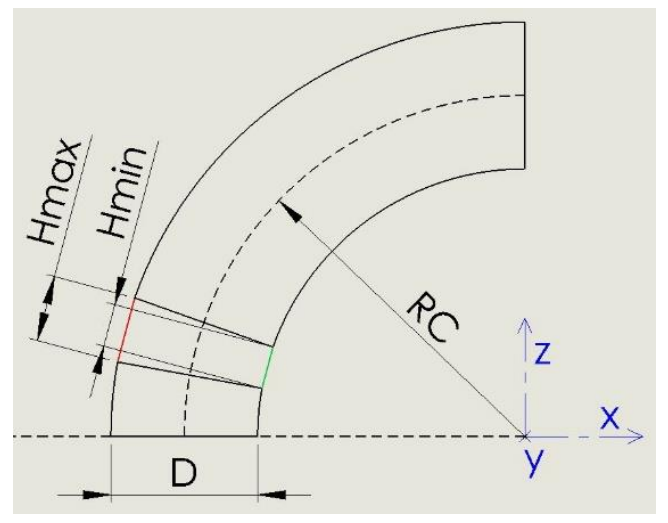

Fig. 4. Distances to calculate the CSratio

$$
\text { CSratio }=R C / D
$$

where: $R C$ is the radius of curvature of the part, and $D$ is the maximum radial size.

For each specific manufacturing process, there is a maximum range or different heights that can be obtained. This means that each process will have a minimum CSratio (calculated with the equation 3 ). In the case of a part with a CSratio below the minimum admissible value, a different approach for defining the shape of the layers is needed.

$$
\text { minCSratio }=\frac{-\frac{H \min }{2 * H \max }-0.5}{\frac{H \min }{H \max }+1}
$$

The suggested solution to this problem consists in incrementing the slicing rotation radius (the one used to create the slicing planes) in the direction of the curvature (Fig. 5), in this way, the admissible region where the desired bead height is inside the regression model gets bigger. An undesired consequence of this is that the part has now a lower radius of curvature than the layers. Thus, at some point, it will be again outside of the model though the limit of the minimum bead height. Another problem is that, due to the part having more curvature that the layers, there is an overhang between two adjacent layers that will increase with time (Fig. 6), which creates a risk of collapse of the deposition material. Thus, one of these previous problems will create a situation where the manufacturing process is not creating an accurate enough part. In that situation, it is possible to create a division of the part, so the rotation axis can be reset and the overhang can be neutralized (Fig. 7). 


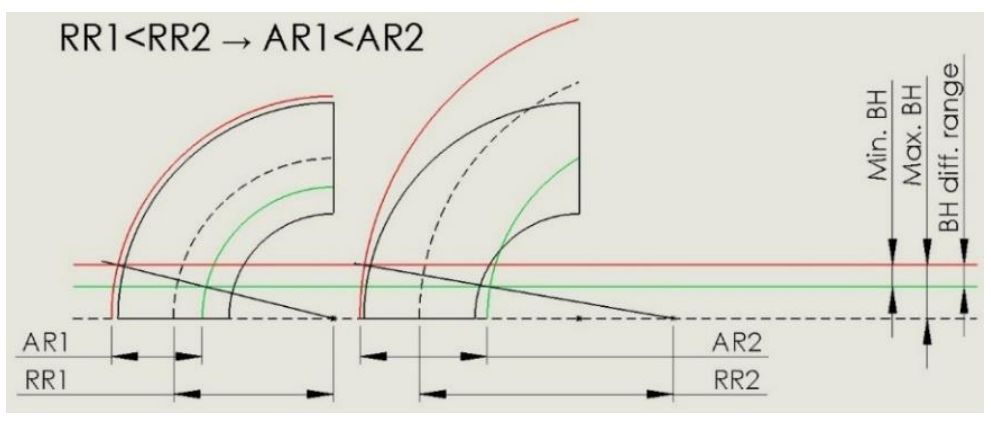

Fig. 5. Increase of rotation radius

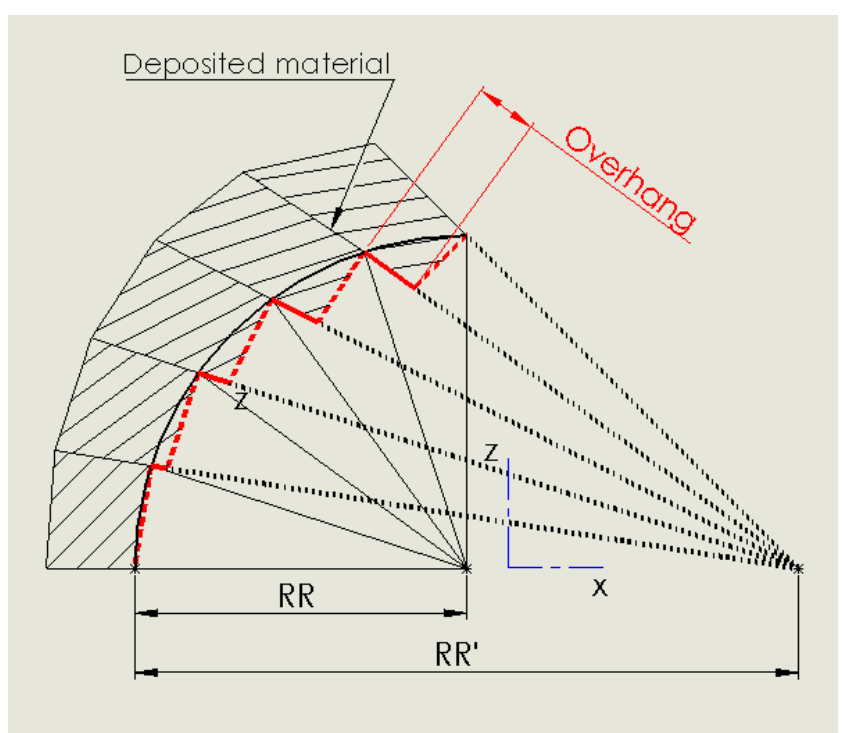

Fig. 6. Overhang due to the increase of rotation radius

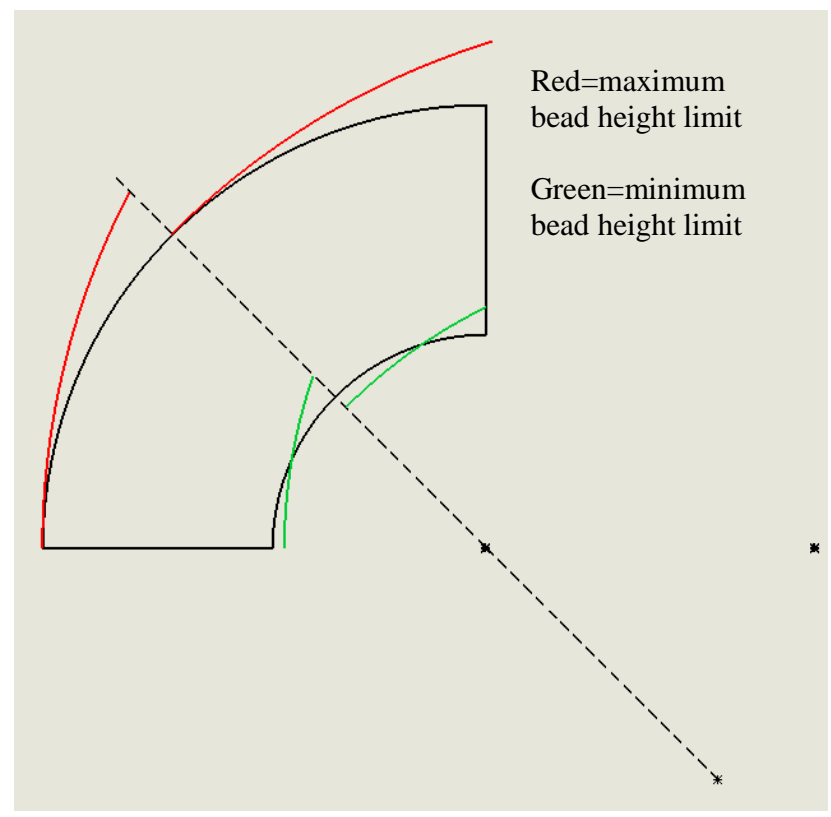

Fig. 7. Part sectioning and rotation axis reset 
This proposed method is controlled by two parameters: the increase of rotation radius and the number of sections. In order to choose the correct parameters for each specific case, an iterative procedure was created with three levels:

- First, check the CSratio of the part, if it is higher than the minimum, the part can be manufactured with variable height layers following its curvature and the iterative procedure is finished; if not, the alternative method will have to be implemented. To start, the lowest value of one section (no sectioning) is chosen in order to avoid/diminish the problems shown in the Querard's approaches [4] related to the sectioning of the part.

- Second, an increase of the rotation radius is chosen. This parameter has to be inside an admissible interval. It is chosen to be big enough for the majority of the desirable bead heights of each point to be inside the regression model (avoiding material accumulation); but it also has to be small enough for the overhang to not reach its maximum value in order to avoid material collapses. The overhang (Fig. 5) can be calculated in function of the rotation radius for each point by means of the projection of each point over the previous slicing plane and its distance to the initial 3D model.

- Last, if no value for the increase of the rotation radius meets the requirements in the previous step, a higher number of sections (+1) has to be fixed, and the iterative procedure starts again. These steps are repeated until all requirements are fulfilled.

\section{TOOLPATH DEFINITION}

In this section, it is shown the suggested procedure for defining a toolpath that can be used to create a program to manufacture complex curved parts with WAAM:

- First, the 3D models of the part sections are sliced with angled planes with the desired angle increment and slicing rotation radius. The sections perimeters are the toolpaths.

- Second, editing the tool axes so they are now perpendicular to each layer of the toolpath (Fig. 8).

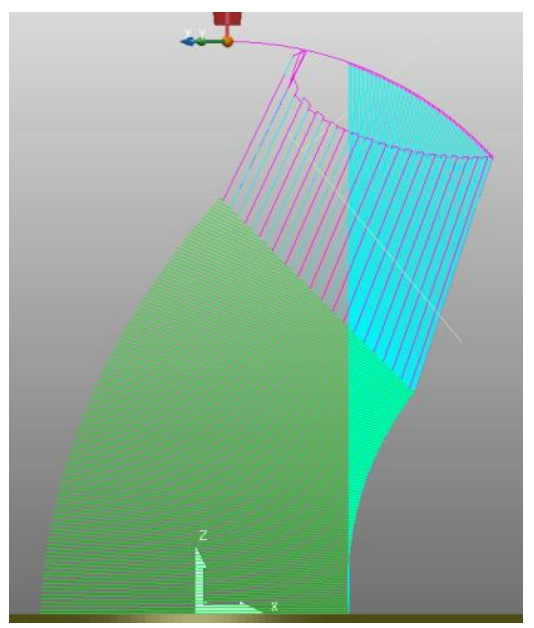

Fig. 8. Final tool axis, part with inadmissible CSratio 
- Finally, the toolpath is simulated with the virtual model of the robot and the G-code is created after checking the possible collisions and singularities. The tool axes must be constricted to be vertical. In this way, the rotary table positioner is forced to incline the part to meet the programmed tool axis (instead of the robot tilting the tool).

\section{TRAVEL SPEEDS DEFINITION}

The definition of the travel speeds for each point is done in a post-editing phase, after creating the original code with constant speed. The distance of each point to the rotation axis (Fig. 9, left) and its desirable bead height (Fig. 9, right) are calculated.

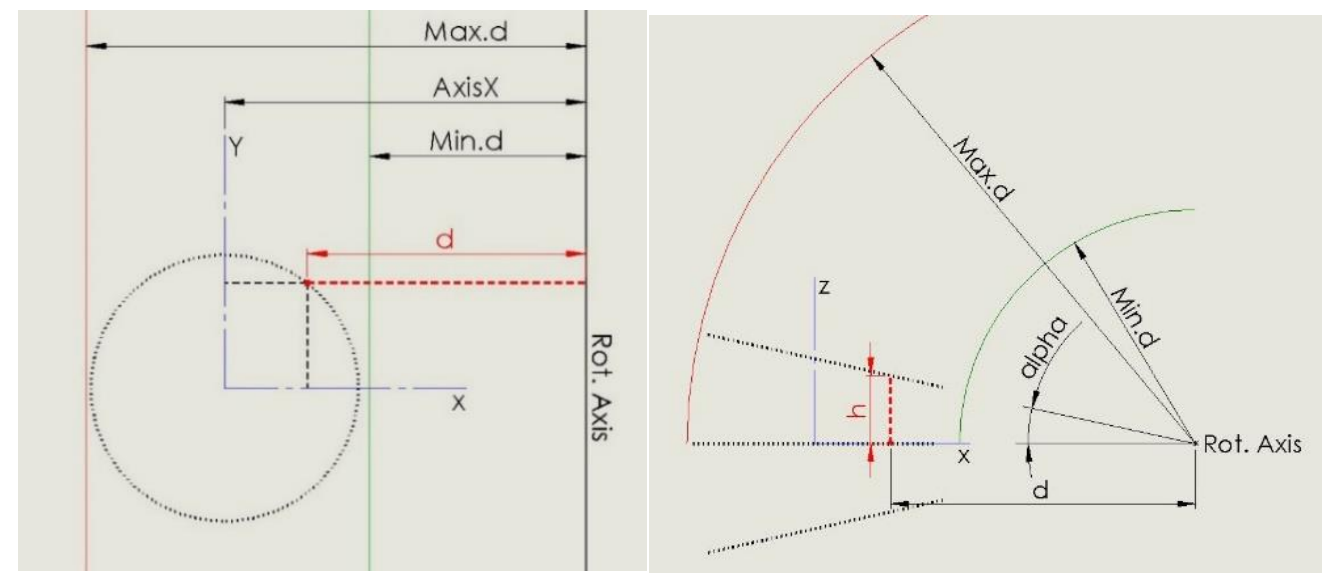

Fig. 9. Distance to the rotation axis and desired bead height

The travel speed for each point is calculated applying the inverse bead height model (equation 1).

\section{USE CASE}

During the geometrical analysis of a use case part manufactured with this method (Fig. 10), it was found that the geometrical accuracy was acceptable on the firsts and medium layers of each section. Some geometrical deviations were found on the last layers of the sections, maximum value of $5.2 \pm 0.1 \mathrm{~mm}$ (relative error of $2.6 \%$ ) between the measured outer surface of the part and the theoretical outer surface of the 3D model. The accuracy of the scanner used is $0.1 \mathrm{~mm}$. These deviations can be partially explained with the thermal deformation of the part during the cooling phase and the dynamic behaviour of the deposition process as the part heats up. A deep study about the monitoring and control of the part temperature over all the phases of the manufacturing process will be needed in order to diminish the impact of the thermal phenomena over the geometrical accuracy of the part, which is necessary to manufacture parts that fulfils the industrial quality standards. 


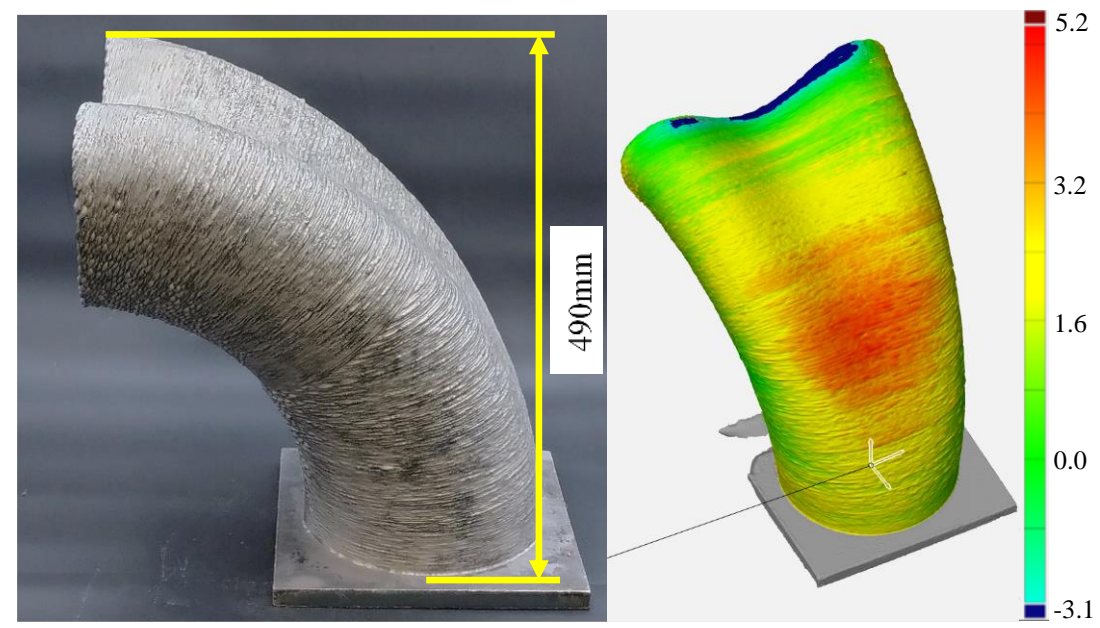

Fig. 10. Use case part manufactured and scanned

\section{CONCLUSIONS}

The manufacturing method suggested in this paper has shown to meet the general objectives described in the section 2 . It has been possible to manufacture steel complex curved parts of high dimensions with this novel methodology without catastrophic events like material collapsing or non-planar deposition, which proves the feasibility of the procedure.

Compared to the other methods of manufacturing curved parts with WAAM shown in the section 1 of this paper, the present one has improved characteristics in terms of surface consistency and applicability. For a part with a CSratio below the minimum admissible for the range of bead height, the suggested method of increasing the slicing rotation radius plus sectioning if needed, will require less sections than the "sectioning" solution, and it will have a more consistent surface finishing than the "variable height plus open layers" solution thanks to the avoidance of open layers. Also, it is a more generalist solution, with a wider range of manufacturable parts than the second approach of pure variable height layers, thanks to the slicing rotation radius increase + sectioning solution proposed in this paper for the case of parts with low CSratio.

\section{REFERENCES}

[1] ALONSO U., VEIGA F., SUÁREZ A., ARTAZA T., 2020, Experimental Investigation of the Influence of Wire Arc Additive Manufacturing on the Machinability of Titanium Parts, Metals, 10, 24.

[2] TABERNERO I., PASKUAL A., ÁlVAREZ P., SUÁREZ A., 2018, Study on Arc Welding Processes for High Deposition Rate Additive Manufacturing, Procedia CIRP, 68, 358-362.

[3] GAO W., ZHANG Y., RAMANUJAN D., RAMANI K., CHEN Y., WILLIAMS C.B., WANG C.C., SHIN Y.C., ZHANG S., ZAVATTIERI P.D., 2015, The Status, Challenges, and Future of Additive Manufacturing in Engineering, Computer-Aided Design, 69, 65-89.

[4] QUERARD V., 2019, Réalisation de Pièces Aéronautiques de Grandes Dimensions Par Fabrication Additive WAAM, Génie mécanique, École centrale de Nantes, https://tel.archives-ouvertes.fr/tel-02389961/, (in French).

[5] DING D., PAN Z., CUIUIR D., LI H., 2015, A Multi-Bead Overlapping Model for Robotic Wire and Arc Additive Manufacturing (WAAM), Robotics and Computer-Integrated Manufacturing, 31, 101-110. 
[6] ISA M., LAZOGLU I., 2019, Five-Axis Additive Manufacturing of Freeform Models Through Buildup of Transition Layers, Journal of Manufacturing Systems, 50, 69-80.

[7] HASCOËT J.Y., QUERARD V., RAUCH M., 2017, Interests of 5 Axis Toolpaths Generation for Wire Arc Additive Manufacturing of Aluminum alloys, Journal of Machine Engineering, 17/3, 51-65.

[8] MUlLER P., HASCOET, J.Y., MOGNOL P., 2014, Toolpaths for Additive Manufacturing of Functionally Graded Materials (FGM) parts, Rapid Prototyping Journal, 20/6, 511-522.

[9] OGINO Y., ASAI S., HIRATA Y., 2018, Numerical Simulation of WAAM Process by a GMAW Weld Pool Model, Welding in the World, 62, 393-401.

[10] DING J., COLEGROVE P., MEHNEN J., et al., 2014, A Computationally Efficient Finite Element Model of Wire and Arc Additive Manufacture, Int. J. Adv. Manuf. Technol., 70, 227-236.

[11] DINOVITZER M., CHEN X., LALIGERTE J., HUANG X., FREI H., 2019, Effect of Wire and Arc Additive Manufacturing (WAAM) Process Parameters on Bead Geometry and Microstructure, Additive Manufacturing, 26, 138-146.

[12] RAGIEZAD M., GHAFFARI M., VAHEDI A., et al., 2019, Microstructural Evolution and Mechanical Properties of a Low-Carbon Low-Alloy Steel Produced by Wire Arc Additive Manufacturing. Int. J. Adv. Manuf. Technol., $105,2121-2134$.

[13] LEI Y., ZENGXI P., DONGHONG D., FENGYANG H., VAN DUIN S., HUIJUN L., WEIHUA L., 2020 , Investigation of Humping Phenomenon for the Multi-Directional Robotic Wire and Arc Additive Manufacturing, Robotics and Computer-Integrated Manufacturing, 63, 101916.

[14] LAM T.F., XIONG Y., DHARMAWAN A.G., et al., 2020, Adaptive Process Control Implementation of Wire Arc Additive Manufacturing for Thin-Walled Components with Overhang Features, Int. J. Adv. Manuf. Technol., 108, 1061-1071.

[15] YILI D., SHENGFU Y., YUNSHENG S., et al., 2018, Wire and Arc Additive Manufacture of High-Building MultiDirectional Pipe Joint, Int. J. Adv. Manuf. Technol., 96, 2389-2396.

[16] XIZHANG C., SU C., WANG Y., et al., 2018, Cold Metal Transfer (CMT) Based Wire and Arc Additive Manufacture (WAAM) System, J. Surf. Investig., 12, 1278-1284.

[17] McNEIL JL, HAMEL WR., PENNEY J., NYCZ A., NOAKES M., 2019, Framework for CAD to Part of Large Scale Additive Manufacturing of Metal (LSAMM) in Arbitrary Direction, Solid Freeform Fabrication Symposium, 1126-1135.

[18] SATISH KUMAR P., SUVARNA RAJU L., SIVA RAMA KRISHNA L., 2020, A Review on Wire Arc Additive Manufacturing (WAAM) Fabricated Components of Ti6AL4V and Steels, International Conference on Emerging Trends in Engineering (ICETE), 2, 587-600.

[19] KNEZOVIĆ N., TOPIĆ A., 2019, Wire and Arc Additive Manufacturing (WAAM) - A New Advance in Manufacturing, International Conference, New Technologies, Development and Applications, 42, 65-71. 